\title{
AN APPLICATION OF THE THEORY OF EDGE SOBOLEV SPACES TO WEAKLY HYPERBOLIC OPERATORS
}

\author{
MICHAEL DREHER \\ Faculty of Mathematics and Computer Sciences \\ Freiberg University of Mining and Technology \\ Agricolastr. 1, 09596 Freiberg, Germany \\ E-mail:dreher@math.tu-freiberg.de
}

1. Introduction. This article reports joint work with Ingo Witt of Potsdam University, Germany, and intends to give an overview on how to apply the theory of edge Sobolev spaces to weakly hyperbolic Cauchy problems. Edge Sobolev spaces are natural spaces for functions living on manifolds with edge type singularity, and have been used successfully in the investigation of elliptic boundary value problems on such manifolds, [8], [15].

The hyperbolic operators considered here are of the form

$$
\begin{aligned}
L=\partial_{t}^{2}+2 \sum_{j=1}^{n} \lambda(t) c_{j}(t) \partial_{t} \partial_{x_{j}}-\sum_{i, j=1}^{n} \lambda(t)^{2} a_{i j}(t) \partial_{x_{i}} \partial_{x_{j}} & \\
& +\sum_{j=1}^{n} \lambda^{\prime}(t) b_{j}(t) \partial_{x_{j}}+c_{0}(t) \partial_{t}
\end{aligned}
$$

where $(t, x) \in \mathbb{R} \times \mathbb{R}^{n}, \partial_{t}=\frac{\partial}{\partial t}, \partial_{x}=\nabla_{x}$, the coefficients $a_{i j}, b_{j}, c_{j} \in C^{\infty}\left(\left[-T_{0}, T_{0}\right], \mathbb{R}\right)$, and $\lambda(t)=t^{l_{*}}$ with some $l_{*} \in \mathbb{N}_{+}=\{1,2,3, \ldots\}$. The operator $L$ is supposed to be (weakly) hyperbolic, i.e., the characteristic roots $\tau=\tau(t, \xi)$, defined as solutions to

$$
\tau^{2}+2 \sum_{j=1}^{n} \lambda(t) c_{j}(t) \tau \xi_{j}-\sum_{i, j=1}^{n} \lambda(t)^{2} a_{i j}(t) \xi_{i} \xi_{j}=0, \quad \xi \in \mathbb{R}^{n}
$$

are real-valued. We assume strict hyperbolicity in case $t \neq 0$. In other words, these roots

2000 Mathematics Subject Classification: Primary 35L80; Secondary 46E35, 35L15.

The paper is in final form and no version of it will be published elsewhere. 
are distinct for $t \neq 0$, more precisely,

$$
\left(\sum_{j=1}^{n} c_{j}(t) \xi_{j}\right)^{2}+\sum_{i, j=1}^{n} a_{i j}(t) \xi_{i} \xi_{j} \geq \alpha_{0}|\xi|^{2}, \quad \alpha_{0}>0, \quad \forall(t, \xi) \in\left[-T_{0}, T_{0}\right] \times \mathbb{R}^{n} .
$$

Hyperbolic operators with coinciding charateristic roots are called weakly hyperbolic, and can exhibit astonishing phenomena not observed in the strictly hyperbolic case. These phenomena depend in a crucial way on the coefficients of the lower order derivatives $\partial_{x_{j}}$. For instance, if the influence of these coefficients is "too large", then the Cauchy problem with initial data prescribed at $t=0$ is not well-posed in $C^{\infty}$. As shown in [9] and [11], necessary and sufficient for the $C^{\infty}$ well-posedness is that the $t$-exponent of these coefficients be at least $l_{*}-1$.

If these so-called Levi conditions are satisfied, then we have well-posedness of (even quasilinear) Cauchy problems in the function spaces $C^{k}\left([0, T], H^{s}\left(\mathbb{R}^{n}\right)\right.$ ) for large $s$ (see [10], [11], [13]) and in $C^{k}\left([0, T], C^{\infty}\left(\mathbb{R}^{n}\right)\right)([2],[4])$. For an interesting counter-example, see [3].

As the simplest possible example, consider the operator

$$
L=\partial_{t}^{2}-t^{2} \partial_{x}^{2}-(4 m+1) \partial_{x}, \quad m \in \mathbb{N} .
$$

In [12], Qi constructed the solution $v=v(t, x)$ to $L v=0, v(0, x)=v_{0}(x), v_{t}(0, x)=0$ :

$$
v(t, x)=\sum_{j=0}^{m} C_{j m} t^{2 j}\left(\partial_{x}^{j} v_{0}\right)\left(x+t^{2} / 2\right), \quad C_{j m} \neq 0 .
$$

This explicit representation shows two facts. First, the solution suffers from a so-called loss of regularity: $v_{0} \in H^{s}(\mathbb{R})$ only implies $v(t, \cdot) \in H^{s-m}(\mathbb{R})$. If $s-m<5 / 2$, then this simple Cauchy problem has no classical solution. Second, singularities of the initial datum $v_{0}$ propagate only to the left. The right characteristic transports no information at all.

We can observe even more phenomena when we prescribe initial data at the line $=-1$, and pass through $t=0$. Singularities will branch, but not always. In [16], Taniguchi and Tozaki studied the Cauchy problem

$$
v_{t t}-t^{2 l_{*}} v_{x x}-b l_{*} t^{l_{*}-1} v_{x}=0, \quad\left(\partial_{t}^{j} v\right)(-1, x)=v_{j}(x), \quad j=0,1,
$$

and assumed that the initial data have a singularity for some $x_{0}$. Since the equation is strictly hyperbolic for $t<0$, this singularity propagates, in general, along each of the two characteristic curves starting at $\left(-1, x_{0}\right)$. When these characteristic curves cross the line $t=0$, they split, and the singularities then propagate along four characteristics for $t>0$. However, in certain cases, determined by a discrete set of values for $b$, one or two of these four characteristic curves do not carry any singularities, see Figure 6 in Section 6 .

The theory of edge Sobolev spaces will enable us to show that semilinear versions of the Taniguchi-Tozaki example behave in the same way.

The linking element between the theory of edge Sobolev spaces and the theory of weakly hyperbolic equations are so-called edge-degenerate differential operators, which are the typical differential operators on manifolds with edge singularities. The operator 
$L$ from (1.1) can be written in the form

$$
L=t^{-2} P\left(t, t \partial_{t}, \Lambda(t) \partial_{x}\right), \quad \Lambda(t)=\int_{0}^{t} \lambda\left(t^{\prime}\right) d t^{\prime},
$$

where $P(t, \tau, \xi)$ is a polynomial in $(\tau, \xi)$ of degree 2 ; and every operator of this form is edge-degenerate.

We will define edge Sobolev spaces $H^{s, \delta ; \lambda}\left((0, T) \times \mathbb{R}^{n}\right)$, where $s \geq 0$ denotes the Sobolev smoothness with respect to $(t, x)$ for $t>0$ and $\delta \in \mathbb{R}$ is an additional parameter. More precisely, the following embeddings are continuous:

$$
H_{\text {comp }}^{s}\left(\mathbb{R}_{+} \times \mathbb{R}^{n}\right) \subset H^{s, \delta ; \lambda}\left((0, T) \times \mathbb{R}^{n}\right) \subset H_{\mathrm{loc}}^{s}\left(\mathbb{R}_{+} \times \mathbb{R}^{n}\right) .
$$

In order to handle the loss of regularity, the elements of the spaces $H^{s, \delta ; \lambda}\left((0, T) \times \mathbb{R}^{n}\right)$ have higher Sobolev smoothness at $t=0$ in the following sense: There are traces $\tau_{j}$, $\tau_{j} u(x)=\left(\partial_{t}^{j} u\right)(0, x)$, with continuous mappings

$$
\tau_{j}: H^{s, \delta ; \lambda}\left((0, T) \times \mathbb{R}^{n}\right) \rightarrow H^{s-\beta j+\beta \delta l_{*}-\beta / 2}\left(\mathbb{R}^{n}\right), \quad \beta=\frac{1}{l_{*}+1}
$$

for all $j \in \mathbb{N}, j<s-1 / 2$.

Roughly speaking, these specially crafted function spaces are able to absorb the loss of regularity, if the parameter $\delta$ is chosen suitably. This will allow to attack semilinear versions of this Cauchy problem by standard fixed point arguments, giving the existence of a solution for small times. Remember that the usual iteration procedure cannot be applied in the case of the standard function space $C\left([0, T], H^{s}\left(\mathbb{R}^{n}\right)\right)$, since we have no longer a mapping which maps this Banach space into itself.

The idea to choose a special function space adapted to the weakly hyperbolic operator has also been used in [5], [6], and [14].

The paper is organized as follows. We briefly sketch the theory of general edge Sobolev spaces in Section 2. In Section 3, the special edge Sobolev spaces $H^{s, \delta ; \lambda}\left((0, T) \times \mathbb{R}^{n}\right)$ are constructed and their properties are presented. Details of the proofs are available in [7]. If we prescribe initial data at $t=-T$, we need so-called double-sided edge Sobolev spaces $H^{s_{+}, s_{-}, \delta_{+}, \delta_{-} ; \lambda}\left((-T, T) \times \mathbb{R}^{n}\right)$, which are defined in Section 4 . The proof of the $H^{s, \delta ; \lambda}\left((0, T) \times \mathbb{R}^{n}\right)$ well-posedness of linear and semilinear Cauchy problems for the operator (1.1) is sketched in Section 5. The details of the proofs can be found in [7]. A semilinar version of the Taniguchi-Tozaki result is given in Section 6. For details, see the beginning of Section 6 .

Finally, we remark that all results presented here are valid for $l_{*}=0$, too. They then coincide with the classical results of the strictly hyperbolic theory.

\section{Differential operators and Sobolev spaces on manifolds with singulari-} ties. In the following, we give a (very) brief overview of the general theory of manifolds with conical and edge singularities, the differential operators acting on such manifolds, and associated Sobolev spaces. This general theory contains much more subjects which are not presented here: manifolds with corners, pseudodifferential operators, ellipticity, parametrices, boundary value problems, index theory, function spaces with discrete or continuous asymptotics, and several others. For sake of brevity, we only consider a very 
simple geometric setting. Detailed expositions can be found in [8] and [15].

2.1. Differential operators and Sobolev spaces on manifolds with conical singularities. Let $Y$ be a closed compact $C^{\infty}$ manifold. We call the set

$$
Y^{\triangle}=\left(\overline{\mathbb{R}_{+}} \times Y\right) /(\{0\} \times Y), \quad \mathbb{R}_{+}=\{t \in \mathbb{R}: t>0\},
$$

a cone with cone basis $Y$, or a manifold with conical singularity, see Figure 1 . This is a customary formulation. Strictly speaking, a manifold with conical singularity is in general no manifold, since it contains the tip of the cone. We hope that this terminology does not confuse.

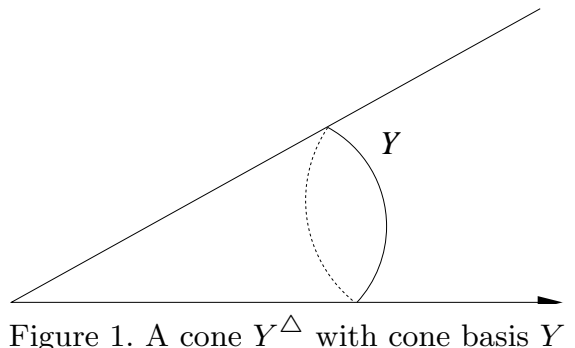

We also introduce the so-called stretched cone $Y^{\wedge}$ with base $Y$ (Figure 2),

$$
Y^{\wedge}=\mathbb{R}_{+} \times Y .
$$

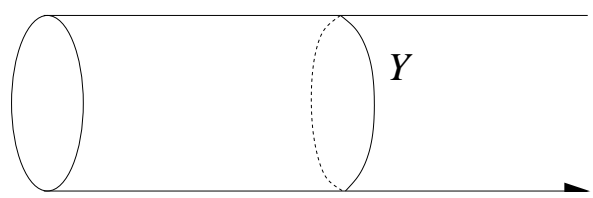

Figure 2. The associated stretched cone $Y^{\wedge}$ with cone basis $Y$

The canonical coordinates on $Y^{\wedge}$ are locally $(t, y)$, where $t$ denotes the distance from the tip of the cone. If $g_{Y}$ denotes a Riemannian metric on $Y$, then

$$
g=d t^{2}+t^{2} g_{Y}
$$

is a Riemannian metric on $Y^{\wedge}$.

The typical $m$-th order differential operator on $Y^{\wedge}$ has then the form

$$
P=t^{-m} \sum_{k=0}^{m} a_{k}(t)\left(t \partial_{t}\right)^{k},
$$

with some coefficients $a_{k} \in C^{\infty}\left(\overline{\mathbb{R}_{+}}, \operatorname{Diff}^{m-k}(Y)\right)$, where $\operatorname{Diff}^{l}(Y)$ denotes the space of all differential operators on $Y$ of order $l$ with $C^{\infty}$ coefficients in local coordinates, which forms a nuclear Fréchet space. The operators of form (2.2) are called Fuchs type operators.

For the definition of Sobolev spaces on manifolds with conical singularities, we introduce the Mellin transform,

$$
\begin{aligned}
& \mathcal{M}: C_{0}^{\infty}\left(\mathbb{R}_{+}\right) \rightarrow \mathcal{A}(\mathbb{C}), \\
& (\mathcal{M} u)(z)=\int_{0}^{\infty} t^{z-1} u(t) d t
\end{aligned}
$$


where $\mathcal{A}$ stands for the analytic functions. The Mellin transform has the inverse transform

$$
\left(\mathcal{M}^{-1} g\right)(t)=\frac{1}{2 \pi i} \int_{\Gamma_{\beta}} t^{-z} g(z) d z
$$

with $\Gamma_{\beta}=\{z \in \mathbb{C}: \operatorname{Re} z=\beta\}$. The crucial property, which links the Mellin transform with Fuchs type operators, is

$$
\mathcal{M}^{-1} z \mathcal{M}=-t \partial_{t}
$$

It is elementary to check that $\left(\mathcal{M}\left(t^{\gamma} u\right)\right)(z)=(\mathcal{M} u)(z+\gamma)$. Then we can also define

$$
\begin{aligned}
& \mathcal{M}_{\gamma}: C_{0}^{\infty}\left(\mathbb{R}_{+}\right) \rightarrow \mathcal{A}(\mathbb{C}), \\
& \left(\mathcal{M}_{\gamma} u\right)(z)=\left(\mathcal{M}\left(t^{-\gamma} u\right)\right)(z+\gamma)
\end{aligned}
$$

which can be extended to a continuous isomorphism

$$
\mathcal{M}_{\gamma}: t^{\gamma} L^{2}\left(\mathbb{R}_{+}\right) \rightarrow L^{2}\left(\Gamma_{1 / 2-\gamma}\right) .
$$

Roughly speaking, the Mellin transform is the composition of the one dimensional Fourier transform and an "exponential stretching of the $t$-variable", in the following sense: Let

$$
\begin{aligned}
& (\mathcal{F} v)(\varrho)=\int_{\mathbb{R}} e^{-i t \varrho} v(t) d t \\
& S_{\gamma}: C_{0}^{\infty}\left(\mathbb{R}_{+}\right) \rightarrow C_{0}^{\infty}(\mathbb{R}) \\
& \left(S_{\gamma} u\right)(t)=\exp \left(-\left(\frac{1}{2}-\gamma\right) t\right) u(\exp (-t))
\end{aligned}
$$

Then we have, for all $\varrho \in \mathbb{R}$,

$$
\left(\mathcal{M}_{\gamma} u\right)\left(\frac{1}{2}-\gamma+i \varrho\right)=\left(\mathcal{F} S_{\gamma} u\right)(\varrho) .
$$

Now we are in a position to define Mellin-Sobolev spaces.

Definition 2.1. By $\mathcal{H}^{s, \gamma}\left(\mathbb{R}_{+}\right), s, \gamma \in \mathbb{R}$, we denote the closure of $C_{0}^{\infty}\left(\mathbb{R}_{+}\right)$under the norm

$$
\|u\|_{\mathcal{H}^{s, \gamma}\left(\mathbb{R}_{+}\right)}^{2}=\frac{1}{2 \pi i} \int_{\Gamma_{1 / 2-\gamma}}\left(1+|z|^{2}\right)^{s}|(\mathcal{M} u)(z)|^{2} d z .
$$

If $s \in \mathbb{N}$ and $\gamma \in \mathbb{R}$, then an equivalent norm is given by

$$
\|u\|_{\mathcal{H}\left(s, \gamma\left(\mathbb{R}_{+}\right)\right.}^{2}=\sum_{k=0}^{s} \int_{0}^{\infty}\left|t^{-\gamma}\left(t \partial_{t}\right)^{k} u(t)\right|^{2} d t
$$

The Mellin-Sobolev spaces form an interpolation scale with respect to the complex interpolation method. We have $\mathcal{H}^{s, \gamma}\left(\mathbb{R}_{+}\right)=t^{\gamma} \mathcal{H}^{s, 0}\left(\mathbb{R}_{+}\right)$and $\mathcal{H}^{0,0}\left(\mathbb{R}_{+}\right)=L^{2}\left(\mathbb{R}_{+}\right)$. In case $s \geq 0$, we have

$$
\mathcal{H}^{0,0}\left(\mathbb{R}_{+}\right) \cap \mathcal{H}^{s, s}\left(\mathbb{R}_{+}\right)=H_{0}^{s}\left(\overline{\mathbb{R}_{+}}\right):=\left\{v \in H^{s}(\mathbb{R}): \operatorname{supp} v \subset \overline{\mathbb{R}_{+}}\right\} .
$$

For completeness, let us give the definition of cone Mellin-Sobolev spaces $\mathcal{H}^{s, \gamma}\left(Y^{\wedge}\right)$.

Definition 2.2. Let $\operatorname{dim} Y=d$. We say that a function $u \in H_{\mathrm{loc}}^{s}\left(Y^{\wedge}\right)$ belongs to $\mathcal{H}^{s, \gamma}\left(Y^{\wedge}\right)$ if for every $\varphi \in C_{0}^{\infty}\left(\overline{Y^{\wedge}}\right)$, supported near some $\{0\} \times\{p\}, p \in Y$, we have

$$
\frac{1}{2 \pi i} \int_{\Gamma_{(d+1) / 2-\gamma}} \int_{\mathbb{R}_{\eta}^{d}}\left(1+|z|^{2}+|\eta|^{2}\right)^{s}\left|\mathcal{M}_{d / 2-\gamma, t \rightarrow z} \mathcal{F}_{y \rightarrow \eta}(\varphi u)(z, \eta)\right|^{2} d \eta d z<\infty,
$$


and if a similar condition for $v(t, y)=t^{-d-1} u\left(t^{-1}, y\right)$ with $-\gamma$ instead of $\gamma$ holds.

Proposition 2.1. If $A$ is a Fuchs type operator of order $m$, then

$$
A: \mathcal{H}^{s, \gamma}\left(Y^{\wedge}\right) \rightarrow \mathcal{H}^{s-m, \gamma-m}\left(Y^{\wedge}\right), \quad s, \gamma \in \mathbb{R} .
$$

If the conical manifold $Y^{\triangle}$ looks as in Figure 3, then it is of course more appropriate to consider function spaces

$$
\mathcal{K}^{s, \gamma}\left(Y^{\wedge}\right)=\omega \mathcal{H}^{s, \gamma}\left(Y^{\wedge}\right)+(1-\omega) H^{s}\left(Y^{\wedge}\right),
$$

where $Y^{\wedge}$ is the associated stretched manifold (see Figure 4), and $\omega \in C_{0}^{\infty}\left(\overline{\mathbb{R}_{+}}\right.$) with $\omega(t) \equiv 1$ near $t=0$. Outside a neighbourhood of the tip of the cone, we should employ the classical Sobolev space $H^{s}\left(Y^{\wedge}\right)$.

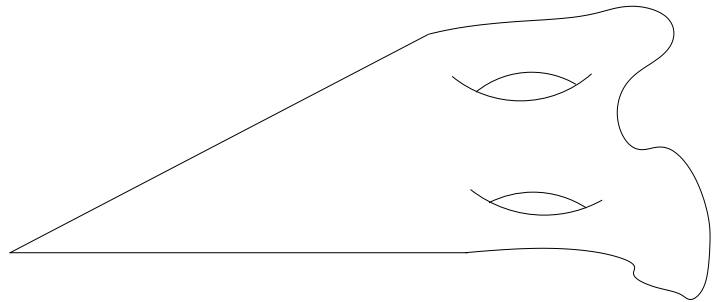

Figure 3. A compact manifold with conical singularity

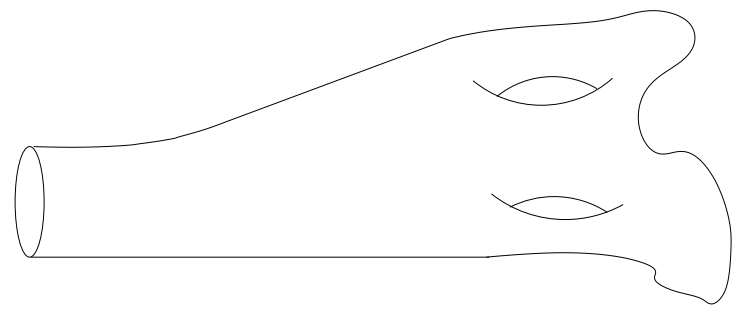

Figure 4. The associated stretched manifold

2.2. Differential operators and Sobolev spaces on manifolds with edge singularities. Let $Y^{\triangle}$ be a manifold with conical singularity as in (2.1), and $Y^{\wedge}$ the associated stretched cone. Then we call

$$
W=Y^{\triangle} \times \mathbb{R}_{x}^{n}, \quad \mathbb{W}=Y^{\wedge} \times \mathbb{R}_{x}^{n}, \quad n \geq 1,
$$

a manifold with edge singularity and a stretched manifold with edge singularity, respectively.

ExAmple 2.1. Take $Y=\{$ point $\}$. Then $Y^{\wedge}=\mathbb{R}_{+}$, and $\mathbb{W}$ is the half-space $\mathbb{R}_{+} \times \mathbb{R}^{n}$ with the edge singularity at $\{0\} \times \mathbb{R}^{n}$.

If $g_{Y}$ is a Riemannian metric on the cone base $Y$, then a Riemannian metric on $\mathbb{W}$ is given by

$$
g=d t^{2}+t^{2} g_{Y}+d x^{2},
$$

and a typical differential operator on $\mathbb{W}$ has the form

$$
P=t^{-m} \sum_{k+|\alpha| \leq m} a_{k, \alpha}(t, x)\left(t \partial_{t}\right)^{k}\left(t \partial_{x}\right)^{\alpha},
$$


where $a_{k, \alpha} \in C^{\infty}\left(\overline{\mathbb{R}_{+}} \times \mathbb{R}_{x}^{n}\right.$, Diff $\left.{ }^{m-k-|\alpha|}(Y)\right)$. Such operators are called edge-degenerate.

Next, we define abstract edge Sobolev spaces.

Definition 2.3. Let $E$ be a Hilbert space, and $\mathcal{S}\left(\mathbb{R}_{x}^{n}, E\right)$ be the Fréchet space of all $E$-valued Schwartz functions. Let $\left\{\kappa_{\nu}\right\}_{\nu>0}$ be a strongly continuous group of isomorphisms, acting on $E$, with $\kappa_{\nu \mu}=\kappa_{\nu} \kappa_{\mu}$ for $\nu, \mu>0$ and $\kappa_{1}=\operatorname{Id}_{E}$. The abstract edge Sobolev space $\mathcal{W}^{s}\left(\mathbb{R}_{x}^{n},\left(E,\left\{\kappa_{\nu}\right\}_{\nu>0}\right)\right)$ is the closure of $\mathcal{S}\left(\mathbb{R}_{x}^{n}, E\right)$ under the norm

$$
\|u\|_{\mathcal{W}^{s}\left(\mathbb{R}_{x}^{n},\left(E,\left\{\kappa_{\nu}\right\}_{\nu>0}\right)\right)}^{2}=\int_{\mathbb{R}_{\xi}^{n}}\langle\xi\rangle^{2 s}\left\|\kappa_{\langle\xi\rangle}^{-1} \hat{u}(\xi)\right\|_{E}^{2} d \xi,
$$

where $\hat{u}(\xi)=\left(\mathcal{F}_{x \rightarrow \xi} u\right)(\xi)$, and $\langle\xi\rangle=\left(1+|\xi|^{2}\right)^{1 / 2}$.

ExAmPLE 2.2. Take $E=H^{s}\left(\mathbb{R}_{t}\right)$, and

$$
\kappa_{\nu} w(t)=\nu^{1 / 2} w(\nu t), \quad t \in \mathbb{R}, \quad \nu>0 .
$$

Then $H^{s}\left(\mathbb{R}_{t} \times \mathbb{R}_{x}^{n}\right)=\mathcal{W}^{s}\left(\mathbb{R}_{x}^{n},\left(H^{s}\left(\mathbb{R}_{t}\right),\left\{\kappa_{\nu}\right\}_{\nu>0}\right)\right)$.

Definition 2.4. Let $s, \gamma \in \mathbb{R}$, and $\left\{\kappa_{\nu}\right\}_{\nu>0}$ as in Example 2.2. The space

$$
\mathcal{W}^{s, \gamma}(\mathbb{W})=\mathcal{W}^{s}\left(\mathbb{R}_{x}^{n},\left(\mathcal{K}^{s, \gamma}\left(Y^{\wedge}\right),\left\{\kappa_{\nu}\right\}_{\nu>0}\right)\right)
$$

is called edge Sobolev space with smoothness $s$ and weight $\gamma$.

Proposition 2.2. If $A$ is an edge-degenerate differential operator of order $m$, then the following map is continuous:

$$
A: \mathcal{W}^{s, \gamma}(\mathbb{W}) \rightarrow \mathcal{W}^{s-m, \gamma-m}(\mathbb{W})
$$

3. Edge Sobolev spaces for weakly hyperbolic operators. The operator $L$ of (1.1) has the form

$$
L=t^{-m} P\left(t, t \partial_{t}, \Lambda(t) \partial_{x}\right), \quad \Lambda(t)=\int_{0}^{t} \lambda\left(t^{\prime}\right) d t^{\prime},
$$

where $P(t, \tau, \xi)$ is a polynomial in $(\tau, \xi)$ of degree $m=2$; hence it is an edge-degenerate operator. This gives rise to the hope that the theory of edge Sobolev spaces could give new insights into weakly hyperbolic Cauchy problems. Actually, the operators of the form (3.1) are a subclass of edge-degenerate differential operators, which is closed under composition. This hints at some modifications in defining cone Sobolev spaces and the group of isomorphisms $\left\{\kappa_{\nu}\right\}_{\nu>0}$.

Definition 3.1. For $s \geq 0, \delta \in \mathbb{R}$, we define a function space

$$
\mathcal{H}_{\sharp}^{s, \delta ; \lambda}\left(\mathbb{R}_{+}\right)=\mathcal{H}^{0, \delta l_{*}}\left(\mathbb{R}_{+}\right) \cap \mathcal{H}^{s, s\left(l_{*}+1\right)+\delta l_{*}}\left(\mathbb{R}_{+}\right)
$$

with smoothness $s$ and weight $\delta$. Let $\omega \in C_{0}^{\infty}\left(\overline{\mathbb{R}_{+}}\right)$be a cut-off function, i.e., $\omega(t) \equiv 1$ near $t=0$. Then the cone Sobolev spaces $H^{s, \delta ; \lambda}\left(\mathbb{R}_{+}\right), H_{0}^{s, \delta ; \lambda}\left(\overline{\mathbb{R}_{+}}\right)$are defined by

$$
\begin{aligned}
& H^{s, \delta ; \lambda}\left(\mathbb{R}_{+}\right)=\omega H^{s}\left(\mathbb{R}_{+}\right)+(1-\omega) \mathcal{H}_{\sharp}^{s, \delta ; \lambda}\left(\mathbb{R}_{+}\right), \\
& H_{0}^{s, \delta ; \lambda}\left(\overline{\mathbb{R}_{+}}\right)=\omega H_{0}^{s}\left(\overline{\mathbb{R}_{+}}\right)+(1-\omega) \mathcal{H}_{\sharp}^{s, \delta ; \lambda}\left(\mathbb{R}_{+}\right) .
\end{aligned}
$$

The space $H^{s, \delta ; \lambda}\left(\mathbb{R}_{+}\right)$has the norm

$$
\|u\|_{H^{s, \delta ; \lambda}\left(\mathbb{R}_{+}\right)}^{2}=\inf \left\{\left\|\omega u_{0}\right\|_{H^{s}\left(\mathbb{R}_{+}\right)}^{2}+\left\|(1-\omega) u_{1}\right\|_{\mathcal{H}_{\sharp}^{s, \delta ; \lambda}\left(\mathbb{R}_{+}\right)}^{2}\right\},
$$


the infimum taken over all $u_{0} \in H^{s}\left(\mathbb{R}_{+}\right), u_{1} \in \mathcal{H}_{\sharp}^{s, \delta ; \lambda}\left(\mathbb{R}_{+}\right)$, with $u=\omega u_{0}+(1-\omega) u_{1}$.

This glueing of different spaces will enable us to treat the loss of regularity, controlled by the parameter $\delta$. Compare also (2.3).

These cone Sobolev spaces have the following properties.

Proposition 3.1.

(a) The spaces $H^{s, \delta ; \lambda}\left(\mathbb{R}_{+}\right)$and $H_{0}^{s, \delta ; \lambda}\left(\overline{\mathbb{R}_{+}}\right)$do not depend on the choice of the cut-off function $\omega$, up to the equivalence of norms.

(b) The restriction of $\mathcal{S}(\mathbb{R})$ to $\overline{\mathbb{R}_{+}}, \mathcal{S}\left(\overline{\mathbb{R}_{+}}\right)$, is dense in $H^{s, \delta ; \lambda}\left(\mathbb{R}_{+}\right)$. If $s \notin 1 / 2+\mathbb{N}$, then $H_{0}^{s, \delta ; \lambda}\left(\overline{\mathbb{R}_{+}}\right)$is the closure of $C_{0}^{\infty}\left(\mathbb{R}_{+}\right)$in $H^{s, \delta ; \lambda}\left(\mathbb{R}_{+}\right)$.

(c) For fixed $\delta \in \mathbb{R},\left\{H^{s, \delta ; \lambda}\left(\mathbb{R}_{+}\right)\right\}_{s \geq 0}$ forms an interpolation scale with respect to the complex interpolation method.

(d) If $l_{*}=0$, then $H^{s, \delta ; \lambda}\left(\mathbb{R}_{+}\right)$and $H_{0}^{s, \delta ; \lambda}\left(\overline{\mathbb{R}_{+}}\right)$coincide with the classical spaces $H^{s}\left(\mathbb{R}_{+}\right)$and $H_{0}^{s}\left(\overline{\mathbb{R}_{+}}\right)$.

A proof is given in [7].

Definition 3.2. For each $\delta \in \mathbb{R}$, we define a group $\left\{\kappa_{\nu}^{(\delta)}\right\}_{\nu>0}$ of isomorphisms by

$$
\kappa_{\nu}^{(\delta)} w(t)=\nu^{\beta / 2-\beta \delta l_{*}} w\left(\nu^{\beta} t\right), \quad \nu>0, \quad t \in \mathbb{R}_{+},
$$

where $\beta=1 /\left(l_{*}+1\right)$. Then we put

$$
\begin{aligned}
& H^{s, \delta ; \lambda}\left(\mathbb{R}_{+} \times \mathbb{R}^{n}\right)=\mathcal{W}^{s}\left(\mathbb{R}_{x}^{n} ;\left(H^{s, \delta ; \lambda}\left(\mathbb{R}_{+}\right),\left\{\kappa_{\nu}^{(\delta)}\right\}_{\nu>0}\right)\right), \\
& H_{0}^{s, \delta ; \lambda}\left(\overline{\mathbb{R}_{+}} \times \mathbb{R}^{n}\right)=\mathcal{W}^{s}\left(\mathbb{R}^{n} ;\left(H_{0}^{s, \delta ; \lambda}\left(\overline{\mathbb{R}_{+}}\right),\left\{\kappa_{\nu}^{(\delta)}\right\}_{\nu>0}\right)\right) .
\end{aligned}
$$

With the use of this definition and Proposition 3.1, the following proposition is easily proved.

Proposition 3.2.

(a) The restriction of $\mathcal{S}\left(\mathbb{R} \times \mathbb{R}^{n}\right)$ to $\overline{\mathbb{R}_{+}} \times \mathbb{R}^{n}, \mathcal{S}\left(\overline{\mathbb{R}_{+}} \times \mathbb{R}^{n}\right)$, is dense in $H^{s, \delta ; \lambda}\left(\mathbb{R}_{+} \times \mathbb{R}^{n}\right)$. If $s \notin 1 / 2+\mathbb{N}$, then $H_{0}^{s, \delta ; \lambda}\left(\overline{\mathbb{R}_{+}} \times \mathbb{R}^{n}\right)$ is the closure of $C_{0}^{\infty}\left(\mathbb{R}_{+} \times \mathbb{R}^{n}\right)$ in $H^{s, \delta ; \lambda}\left(\mathbb{R}_{+} \times \mathbb{R}^{n}\right)$.

(b) For every fixed $\delta \in \mathbb{R},\left\{H^{s, \delta ; \lambda}\left(\mathbb{R}_{+} \times \mathbb{R}^{n}\right)\right\}_{s \geq 0}$ forms an interpolation scale with respect to the complex interpolation method.

(c) If $l_{*}=0$, then the spaces $H^{s, \delta ; \lambda}\left(\mathbb{R}_{+} \times \mathbb{R}^{n}\right)$ and $H_{0}^{s, \delta ; \lambda}\left(\overline{\mathbb{R}_{+}} \times \mathbb{R}^{n}\right)$ coincide with the classical spaces $H^{s}\left(\mathbb{R}_{+} \times \mathbb{R}^{n}\right)$ and $H_{0}^{s}\left(\overline{\mathbb{R}_{+}} \times \mathbb{R}^{n}\right)$, respectively.

(d) We have the continuous embeddings

$$
H_{\text {comp }}^{s}\left(\mathbb{R}_{+} \times \mathbb{R}^{n}\right) \subset H^{s, \delta ; \lambda}\left(\mathbb{R}_{+} \times \mathbb{R}^{n}\right) \subset H_{\text {loc }}^{s}\left(\mathbb{R}_{+} \times \mathbb{R}^{n}\right) .
$$

The functions of $H^{s, \delta ; \lambda}\left(\mathbb{R}_{+} \times \mathbb{R}^{n}\right)$ have $H^{s}$-smoothness away from $t=0$. As the following result shows, these functions will be smoother at $t=0$ if $\delta \in \mathbb{R}$ is not too small.

Proposition 3.3. Let $s>1 / 2, \delta \in \mathbb{R}$. Then, for each $j \in \mathbb{N}, j<s-1 / 2$, the map $\mathcal{S}\left(\overline{\mathbb{R}_{+}} \times \mathbb{R}^{n}\right) \rightarrow \mathcal{S}\left(\mathbb{R}^{n}\right), u \mapsto\left(\partial_{t}^{j} u\right)(0, x)$, extends by continuity to a map

$$
\tau_{j}: H^{s, \delta ; \lambda}\left(\mathbb{R}_{+} \times \mathbb{R}^{n}\right) \rightarrow H^{s-\beta j+\beta \delta l_{*}-\beta / 2}\left(\mathbb{R}^{n}\right) .
$$

Furthermore, the map

$$
H^{s, \delta ; \lambda}\left(\mathbb{R}_{+} \times \mathbb{R}^{n}\right) \rightarrow \prod_{j<s-1 / 2} H^{s-\beta j+\beta \delta l_{*}-\beta / 2}\left(\mathbb{R}^{n}\right), u \mapsto\left\{\tau_{j} u\right\}_{j<s-1 / 2}
$$


is surjective.

Finally, we describe mapping properties of the usual differential operators.

Proposition 3.4. For $s \geq 0, \delta \in \mathbb{R}$, the following maps are continuous:

(a) $t^{l} \partial_{t}^{k}: H^{s+k, \delta ; \lambda}\left(\mathbb{R}_{+} \times \mathbb{R}^{n}\right) \rightarrow H^{s, \delta+l / l_{*} ; \lambda}\left(\mathbb{R}_{+} \times \mathbb{R}^{n}\right)$ for $l=0,1, \ldots, l_{*}$;

(b) $\varphi(t) \partial_{x}^{\alpha}: H^{s+|\alpha|, \delta ; \lambda}\left(\mathbb{R}_{+} \times \mathbb{R}^{n}\right) \rightarrow H^{s, \delta ; \lambda}\left(\mathbb{R}_{+} \times \mathbb{R}^{n}\right)$ for $\varphi=\varphi(t) \in \mathcal{S}\left(\overline{\mathbb{R}_{+}}\right)$.

The functions of $H^{s, \delta ; \lambda}\left(\mathbb{R}_{+} \times \mathbb{R}^{n}\right)$ can have power type growth for $t \rightarrow \infty$. In other words, these spaces are not closed under point-wise multiplication, even for large $s$. Since we are anyway only interested in solutions to hyperbolic Cauchy problems for finite time intervals $[0, T]$, we set

$$
H^{s, \delta ; \lambda}\left((0, T) \times \mathbb{R}^{n}\right)=\left.H^{s, \delta ; \lambda}\left(\mathbb{R}_{+} \times \mathbb{R}^{n}\right)\right|_{(0, T) \times \mathbb{R}^{n}}
$$

where $0<T \leq T_{0}$, with some fixed $T_{0}$, and equip this space with its infimum norm. An equivalent norm can be given in case $s \in \mathbb{N}$ :

Lemma 3.1. Let $s \in \mathbb{N}, \delta \in \mathbb{R}$ and $T>0$. Then the infimum norm of the space $H^{s, \delta ; \lambda}\left((0, T) \times \mathbb{R}^{n}\right)$ is equivalent to the norm $\|\cdot\|_{s, \delta ; T}$, which is defined as

$$
\begin{aligned}
\|u\|_{s, \delta ; T}^{2} & =\sum_{l=0}^{s} T^{2 l-1} \int_{0}^{T} \int_{\mathbb{R}_{\xi}^{n}} \vartheta_{l}(t, \xi)^{2}\left|\partial_{t}^{l} \hat{u}(t, \xi)\right|^{2} d \xi d t, \\
\vartheta_{l}(t, \xi) & = \begin{cases}\langle\xi\rangle^{s-l} \lambda\left(t_{\xi}\right)^{-\delta-l}, & 0 \leq t \leq t_{\xi}, \\
\langle\xi\rangle^{s-l} \lambda(t)^{-\delta-l}, & t_{\xi} \leq t \leq T .\end{cases}
\end{aligned}
$$

Here we have introduced the notation $t_{\xi}=\langle\xi\rangle^{-\beta}, \beta=1 /\left(l_{*}+1\right)$.

These weighted norms and an elementary, but long, calculation shows the completeness under pointwise multiplication for large integer $s$ :

Proposition 3.5. Suppose that $s \in \mathbb{N}, s+\delta \geq 0$, and $\min \left\{s, s+\beta \delta l_{*}\right\}>(n+2) / 2$. Then $H^{s, \delta ; \lambda}\left((0, T) \times \mathbb{R}^{n}\right)$ is an algebra under pointwise multiplication uniformly in $T$. This means

$$
\|u v\|_{s, \delta ; T} \leq C\|u\|_{s, \delta ; T}\|v\|_{s, \delta ; T}, \quad \forall u, v \in H^{s, \delta ; \lambda}\left((0, T) \times \mathbb{R}^{n}\right),
$$

where $C$ does not depend on $T, 0<T \leq T_{0}$.

The two conditions $s>(n+2) / 2$ and $s+\beta \delta l_{*}>(n+2) / 2$ are related to the two different Sobolev regularities of the elements of $H^{s, \delta ; \lambda}\left(\mathbb{R}_{+} \times \mathbb{R}^{n}\right)$, see Propositions 3.2 and 3.3.

Interpolating with respect to $s$ (see [1]) one can derive

Proposition 3.6. Let $f=f(u)$ be entire with $f(0)=0$, i.e., $f(u)=\sum_{j=1}^{\infty} f_{j} u^{j}$ for all $u \in \mathbb{R}$. Suppose $\lfloor s\rfloor+\delta \geq 0$ and $\min \left\{\lfloor s\rfloor,\lfloor s\rfloor+\beta \delta l_{*}\right\}>(n+2) / 2$, where $\lfloor s\rfloor$ denotes the integer part of $s$. Then we have the estimates

$$
\begin{aligned}
\|f(u)\|_{s, \delta ; T} & \leq C_{1}(R)\|u\|_{s, \delta ; T}, \\
\|f(u)-f(v)\|_{s, \delta ; T} & \leq C_{1}(R)\|u-v\|_{s, \delta ; T}
\end{aligned}
$$

for $u, v \in H^{s, \delta ; \lambda}\left((0, T) \times \mathbb{R}^{n}\right)$ with $\|u\|_{s, \delta ; T} \leq R,\|v\|_{s, \delta ; T} \leq R$.

We conclude this section with an embedding lemma. 
Lemma 3.2. For $s, s^{\prime} \geq 0, \delta, \delta^{\prime} \in \mathbb{R}$, and $T>0$, the embedding

$$
H^{s, \delta ; \lambda}\left((0, T) \times \mathbb{R}^{n}\right) \subseteq H^{s^{\prime}, \delta^{\prime} ; \lambda}\left((0, T) \times \mathbb{R}^{n}\right)
$$

is continuous if and only if $s \geq s^{\prime}$ as well as $s+\beta \delta l_{*} \geq s^{\prime}+\beta \delta^{\prime} l_{*}$.

4. Double-sided edge Sobolev spaces. The specially constructed edge Sobolev spaces allow the discussion of weakly hyperbolic equations for $t \geq 0$. For the double-sided case, $-T \leq t \leq T$, we glue together two copies of such spaces at the line $t=0$ :

Definition 4.1. Let $s \geq 0, \delta \in \mathbb{R}$. We say that $u \in H^{s, \delta ; \lambda}\left((-T, T) \times \mathbb{R}^{n}\right)$ if $u(t, x) \in$ $H^{s, \delta ; \lambda}\left((0, T) \times \mathbb{R}^{n}\right), u(-t, x) \in H^{s, \delta ; \lambda}\left((0, T) \times \mathbb{R}^{n}\right)$, and $u(t, x)-u(-t, x) \in H_{0}^{s, \delta ; \lambda}((0, T) \times$ $\left.\mathbb{R}^{n}\right)$.

The last condition means that the traces at $t=0$ taken from $\{t>0\}$ and $\{t<0\}$ coincide. The functions of these spaces have the same Sobolev regularity for $t<0$ and $t>0$. This is quite strong a condition; it is more natural to only assume equal smoothness for $t=+0$ and $t=-0$, see Proposition 3.3.

Definition 4.2. Let $s_{-}, s_{+} \geq 0, \delta_{-}, \delta_{+} \in \mathbb{R}$ and suppose that

$$
s_{-}+\beta \delta_{-} l_{*}=s_{+}+\beta \delta_{+} l_{*}, \quad s_{+} \leq s_{-} .
$$

We say that $u \in H^{s_{-}, s_{+}, \delta_{-}, \delta_{+} ; \lambda}\left((-T, T) \times \mathbb{R}^{n}\right)$ if $u \in H^{s_{+}, \delta_{+} ; \lambda}\left((-T, T) \times \mathbb{R}^{n}\right)$ and $u(-t, x) \in H^{s_{-}, \delta_{-} ; \lambda}\left((0, T) \times \mathbb{R}^{n}\right)$. The norm is given by

$$
\begin{aligned}
\|u(t, x)\|_{H^{s_{-}, s_{+}, \delta_{-}, \delta_{+} ; \lambda}}^{2}\left((-T, T) \times \mathbb{R}^{n}\right) & \\
& =\|u(t, x)\|_{H^{s_{+}, \delta_{+} ; \lambda}\left((0, T) \times \mathbb{R}^{n}\right)}^{2}+\|u(-t, x)\|_{H^{s_{-}, \delta_{-} ; \lambda}\left((0, T) \times \mathbb{R}^{n}\right)}^{2} .
\end{aligned}
$$

We are allowed to define the norm this way because Lemma 3.2 implies the continuous embedding $H^{s_{-}, \delta_{-} ; \lambda}\left((0, T) \times \mathbb{R}^{n}\right) \subset H^{s_{+}, \delta_{+} ; \lambda}\left((0, T) \times \mathbb{R}^{n}\right)$.

Such function spaces enable us to consider functions with Sobolev regularities $s_{-}>$ $s_{-}+\beta \delta_{-} l_{*}>s_{+}$in the regions $\{t<0\},\{t=0\},\{t>0\}$, respectively. The following example shows that such situations really occur.

Example 4.1. Consider the operator of Taniguchi and Tozaki,

$$
L=\partial_{t}^{2}-t^{2 l_{*}} \partial_{x}^{2}-b l_{*} t^{l_{*}-1} \partial_{x}, \quad x \in \mathbb{R}, \quad b \in \mathbb{R}, \quad l_{*} \in \mathbb{N}_{+} .
$$

In [16], the solution $v(t, x)$ to the Cauchy problem

$$
L v=0, \quad\left(\partial_{t}^{j} v\right)(0, x)=v_{j}(x), \quad j=0,1,
$$

was expressed in the form

$$
\begin{aligned}
\hat{v}(t, \xi)=\exp (-i \Lambda(t) \xi)_{1} \mathrm{~F}_{1}\left(\beta(1+b) l_{*} / 2, \beta l_{*}, 2 i \Lambda(t) \xi\right) & \hat{v}_{0}(\xi) \\
& +t \exp (-i \Lambda(t) \xi)_{1} \mathrm{~F}_{1}\left(\beta(1+b) l_{*} / 2+\beta, \beta\left(l_{*}+2\right), 2 i \Lambda(t) \xi\right) \hat{v}_{1}(\xi),
\end{aligned}
$$

where $\Lambda(t)=t^{l_{*}+1} /\left(l_{*}+1\right)$, and ${ }_{1} \mathrm{~F}_{1}(\alpha, \gamma, z)$ is the confluent hypergeometric function with the asymptotic behaviour

$$
{ }_{1} \mathrm{~F}_{1}(\alpha, \gamma, z)=\frac{\Gamma(\gamma)}{\Gamma(\gamma-\alpha)} e^{ \pm i \pi \alpha} z^{-\alpha}\left(1+\mathcal{O}\left(|z|^{-1}\right)\right)+\frac{\Gamma(\gamma)}{\Gamma(\alpha)} e^{z} z^{\alpha-\gamma}\left(1+\mathcal{O}\left(|z|^{-1}\right)\right)
$$


for $|z| \rightarrow \infty$. If we fix $t \neq 0$, and introduce the notation

$$
\begin{array}{ll}
\alpha_{0}^{-}=-\beta(1+b) l_{*} / 2, & \alpha_{0}^{+}=-\beta(1-b) l_{*} / 2, \\
\alpha_{1}^{-}=\alpha_{0}^{-}-\beta, & \alpha_{1}^{+}=\alpha_{0}^{+}-\beta,
\end{array}
$$

then we have, for $|\xi| \rightarrow \infty$, the asymptotic behaviour

$$
\begin{aligned}
\hat{v}(t, \xi)=(\exp (-i \Lambda(t) \xi) & \left.\mathcal{O}\left(|\xi|^{\alpha_{0}^{-}}\right)+\exp (i \Lambda(t) \xi) \mathcal{O}\left(|\xi|^{\alpha_{0}^{+}}\right)\right) \hat{v}_{0}(\xi) \\
& +\left(\exp (-i \Lambda(t) \xi) \mathcal{O}\left(|\xi|^{\alpha_{1}^{-}}\right)+\exp (i \Lambda(t) \xi) \mathcal{O}\left(|\xi|^{\alpha_{1}^{+}}\right)\right) \hat{v}_{1}(\xi)
\end{aligned}
$$

In general, one of the exponents $\alpha_{0}^{-}, \alpha_{0}^{+}$and one of the exponents $\alpha_{1}^{-}, \alpha_{1}^{+}$is positive, the other one is negative. We observe a loss of $\max \left\{\alpha_{0}^{-}, \alpha_{0}^{+}\right\}$derivatives when we start from $t=0$ in positive or negative direction, and a loss of $\max \left\{-\alpha_{0}^{-},-\alpha_{0}^{+}\right\}$derivatives when we arrive at $t=0$, coming from $t<0$ or $t>0$.

5. Weakly hyperbolic Cauchy problems. We apply the theory of edge Sobolev spaces to weakly hyperbolic equations, starting with the Cauchy problem

$$
L w(t, x)=f(t, x), \quad\left(\partial_{t}^{j} w\right)(0, x)=w_{j}(x), \quad j=0,1,
$$

where $0 \leq t \leq T_{0}$. To discuss existence and regularity of the solution, we denote by $\hat{w}(t, \xi)$ the partial Fourier transform of $w$ and introduce the weight function

$$
g(t, \xi)=\omega(\Lambda(t)\langle\xi\rangle) t_{\xi}^{-1}+(1-\omega(\Lambda(t)\langle\xi\rangle)) \lambda(t)|\xi|, \quad(t, \xi) \in\left[0, T_{0}\right] \times \mathbb{R}^{n},
$$

where $\omega \in C^{\infty}\left(\overline{\mathbb{R}_{+}}\right)$is a cut-off function which is identically equal to 1 in a neighbourhood of the origin. Then we obtain the first order system

$$
\begin{aligned}
& D_{t} W(t, \xi)=A(t, \xi) W(t, \xi)+F(t, \xi), \\
& A(t, \xi)=\left(\begin{array}{cc}
\left(D_{t} g(t, \xi)\right) / g(t, \xi) & g(t, \xi) \\
\left(\lambda(t)^{2}|\xi|^{2} a(t, \xi)-i \lambda^{\prime}(t)|\xi| b(t, \xi)\right) / g(t, \xi) & -2 c(t, \xi) \lambda(t)|\xi|+i c_{0}(t)
\end{array}\right), \\
& W(t, \xi)={ }^{t}\left(g(t, \xi) \hat{w}(t, \xi), D_{t} \hat{w}(t, \xi)\right), \\
& F(t, \xi)={ }^{t}(0,-\hat{f}(t, \xi))
\end{aligned}
$$

where we have set $D_{t}=-i \partial_{t}$, and

$$
a(t, \xi)=\sum_{i, j=1}^{n} a_{i j}(t) \frac{\xi_{i} \xi_{j}}{|\xi|^{2}}, \quad b(t, \xi)=-\sum_{j=1}^{n} b_{j}(t) \frac{\xi_{j}}{|\xi|}, \quad c(t, \xi)=\sum_{j=1}^{n} c_{j}(t) \frac{\xi_{j}}{|\xi|} .
$$

The existence and uniqueness of $W(t, \xi)$ as a solution to a linear system of ordinary differential equations is clear. It remains to study the regularity of $w(t, x)$, or the decay of $W(t, \xi)$ for $|\xi| \rightarrow \infty$. Therefore we consider the fundamental matrix $X\left(t, t^{\prime}, \xi\right)$,

$$
D_{t} X\left(t, t^{\prime}, \xi\right)=A(t, \xi) X\left(t, t^{\prime}, \xi\right), \quad X(t, t, \xi)=I, \quad 0 \leq t^{\prime}, t \leq T_{0},
$$

which is connected to $W$ via $W(t, \xi)=X\left(t, t^{\prime}, \xi\right) W\left(t^{\prime}, \xi\right)+i \int_{t^{\prime}}^{t} X\left(t, t^{\prime \prime}, \xi\right) F\left(t^{\prime \prime}, \xi\right) d t^{\prime \prime}$.

The following lemma is the key to the estimate of $W$. 
LEMMA 5.1. There is a constant $C>0$ such that

$$
\begin{aligned}
& \left\|X\left(t, t^{\prime}, \xi\right)\right\| \leq C\left(\frac{g(t, \xi)}{g\left(t^{\prime}, \xi\right)}\right)^{Q_{0}+1}, \quad 0 \leq t^{\prime} \leq t \leq T_{0}, \\
& \left\|X\left(t, t^{\prime}, \xi\right)\right\| \leq C\left(\frac{g(t, \xi)}{g\left(t^{\prime}, \xi\right)}\right)^{-Q_{0}}, \quad 0 \leq t \leq t^{\prime} \leq T_{0},
\end{aligned}
$$

holds for all $\xi \in \mathbb{R}^{n}$, where $Q_{0}$ is defined as

$$
Q_{0}=-\frac{1}{2}+\sup _{\xi} \frac{|b(0, \xi)+c(0, \xi)|}{2 \sqrt{c(0, \xi)^{2}+a(0, \xi)}} .
$$

A proof can be found in [7].

Assuming $s \in \mathbb{N}_{+}$, we can then employ the equivalent norms $\|\cdot\|_{s, Q ; T}$ defined in Lemma 3.1, and deduce the following a priori estimate after a direct, but long, calculation.

Proposition 5.1. Let $s \in \mathbb{N}_{+}, Q \geq Q_{0}, A=\beta Q l_{*}$, and assume $w_{j} \in H^{s+A-\beta j}\left(\mathbb{R}^{n}\right)$, $j=0,1$, and $f \in H^{s-1, Q+1 ; \lambda}\left((0, T) \times \mathbb{R}^{n}\right)$. Then the solution $w$ to (5.1) satisfies the estimate

$$
\|w\|_{s, Q ; T} \leq C_{0}\left(\left\|w_{0}\right\|_{H^{s+A}\left(\mathbb{R}^{n}\right)}+\left\|w_{1}\right\|_{H^{s+A-\beta}\left(\mathbb{R}^{n}\right)}+T\|f\|_{s-1, Q+1 ; T}\right)
$$

for all $0<T \leq T_{0}$. The constant $C_{0}$ depends on $s$ and $Q$, but not on $T$.

The parameter $A$ equals the number of lost derivatives, when we pass from $\{t=0\}$ to $\{t>0\}$. Example 4.1 shows that the lower bound $A_{0}=\beta Q_{0} l_{*}$ for $A$ cannot be chosen smaller.

REMARK 5.1. By interpolation in $s$, we can easily relax the assumption " $s \in \mathbb{N}_{+}$" in Proposition 5.1 to " $s \in \mathbb{R}, s \geq 1$ ".

Exploiting Proposition 3.6 and the a priori estimate of Proposition 5.1, the wellposedness of the Cauchy problem

$$
L u=f(u), \quad\left(\partial_{t}^{j} u\right)(0, x)=u_{j}(x), \quad j=0,1,
$$

for small time intervals $[0, T]$ can be proved by standard fixed point arguments.

Theorem 1. Let $s \in \mathbb{N}$ and assume that $\min \left\{s, s+\beta Q_{0} l_{*}\right\}>(n+2) / 2$, where $Q_{0}$ be the number from (5.6). Suppose that $f=f(u)$ is entire with $f(0)=0$. Let $Q \geq Q_{0}$ and $A=\beta Q l_{*}$. Then, for $u_{j} \in H^{s+A-j \beta}\left(\mathbb{R}^{n}\right), j=0,1$, a small number $T>0$ can be found in such a way that there is a uniquely determined solution $u \in H^{s, Q ; \lambda}\left((0, T) \times \mathbb{R}^{n}\right)$ to the Cauchy problem (5.7).

6. Branching of singularities for semilinear equations. How do singularities of initial data propagate for nonlinear nonstrictly hyperbolic equations?

In opposite to the strictly hyperbolic case, the characteristics of a weakly hyperbolic operator may intersect tangentially, and surprising phenomena can happen, even in the linear case. For an equation

$$
v_{t t}-t^{2 l_{*}} v_{x x}-b l_{*} t^{l_{*}-1} v_{x}=0
$$

with initial data prescribed at $t=0$ or $t=-1$, respectively, the explicit representation of the solution by means of special functions (see Example 4.1) enables us to show that 
singularities of the initial data can propagate as shown in Figures 5 and 6 , respectively. Depending on the parameter $b$ in a subtle way, some branch (dotted line) carries no singularities (see [12], [16]).

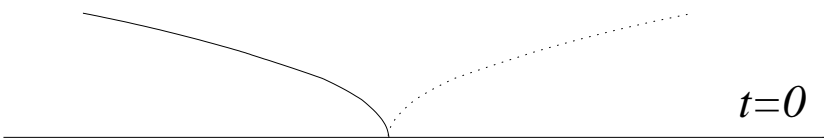

Figure 5. The example of Qi

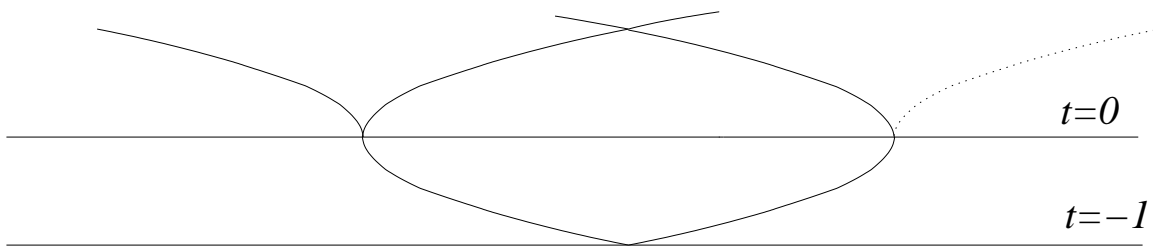

Figure 6 . The example of Taniguchi and Tozaki

The results of this section will tell us that the same pictures can be drawn in the semilinear case, that is

$$
u_{t t}-t^{2 l_{*}} u_{x x}-b l_{*} t^{l_{*}-1} u_{x}=f(u) .
$$

The main difficulty here is the loss of regularity. More precisely, there are two losses: the first, when we arrive at $t=0$, coming from $t<0$; and the second, when we start from $t=0$ and pass to $t>0$. (Moreover, another loss of regularity would occur when we started at $t=0$ and went backwards in time, compare Example 4.1.) It is not obvious how these two losses interact with the nonlinearity $f=f(u)$.

The edge Sobolev spaces $H^{s_{-}, s_{+}, \delta_{-}, \delta_{+} ; \lambda}\left((-1,1) \times \mathbb{R}^{n}\right)$ seem to be an optimal tool for handling this problem, because they are able to absorb all these losses of regularity provided that the parameters $s_{ \pm}, \delta_{ \pm}$are chosen carefully. Furthermore, they are algebras if the smoothness parameters $s_{ \pm}$are large enough; hence the superposition $f=f(u)$ maps these spaces into themselves if $f$ is entire.

This way, it can be deduced that $u$ belongs to the same edge Sobolev space as $v$, cf. Proposition 5.1. If we are able to prove that $u-v$ has higher regularity than $v$, then we have shown that $u$ and $v$ share the same singularity transporting characteristics.

THEOREM 2. Let s satisfy the assumptions of Theorem 1 , and $u_{j} \in H^{s+\beta Q_{0} l_{*}-\beta j}\left(\mathbb{R}^{n}\right)$, $j=0,1$, where $Q_{0}$ is given by (5.6). Let $u, v \in H^{s, Q_{0} ; \lambda}\left((0, T) \times \mathbb{R}^{n}\right)$ be the solutions to

$$
\begin{aligned}
& L u=f(u),\left(\partial_{t}^{j} u\right)(0, x)=u_{j}(x), j=0,1, \\
& L v=0, \quad\left(\partial_{t}^{j} v\right)(0, x)=u_{j}(x), j=0,1 .
\end{aligned}
$$

Then the difference $u-v$ belongs to $H^{s+\beta, Q_{0} ; \lambda}\left((0, T) \times \mathbb{R}^{n}\right)$.

Proof. From Proposition 3.6 we obtain $f(u) \in H^{s, Q_{0} ; \lambda}\left((0, T) \times \mathbb{R}^{n}\right)$. The embedding lemma 3.2 then yields $f(u) \in H^{s-1+\beta, Q_{0}+1 ; \lambda}\left((0, T) \times \mathbb{R}^{n}\right)$. The difference $u-v$ solves $L(u-v)=f(u)$ and has vanishing initial data for $t=0$. We apply Remark 5.1 and the proof is complete. 
ExAmple 6.1. Consider Qi's operator $L$ from (1.2). Then $l_{*}=1, \beta=1 / 2$, and $Q_{0}=2 m$. Remark 5.1, and Theorems 1, 2 imply that the solutions $u, v$ satisfy

$$
u, v \in H^{s, 2 m ; \lambda}((0, T) \times \mathbb{R}), \quad u-v \in H^{s+1 / 2,2 m ; \lambda}((0, T) \times \mathbb{R})
$$

provided that $u_{0} \in H^{s+m}(\mathbb{R}), u_{1} \in H^{s+m-1 / 2}(\mathbb{R})$. Proposition 3.2 then yields

$$
u, v \in H_{\mathrm{loc}}^{s}((0, T) \times \mathbb{R}), \quad u-v \in H_{\mathrm{loc}}^{s+1 / 2}((0, T) \times \mathbb{R}) .
$$

The explicit representation (1.3) of $v$ confirms that our statements on the smoothness of $v$ are exact. We find that the strongest singularities of $u$ coincide with the singularities of $v$. The latter can be looked up in (1.3) in case $u_{1} \equiv 0$.

Now we consider the Cauchy problems

$$
\begin{aligned}
& L u=f(u), \quad\left(\partial_{t}^{j} u\right)(-1, x)=\varepsilon u_{j}(x), \quad j=0,1, \\
& L v=0, \quad\left(\partial_{t}^{j} v\right)(-1, x)=\varepsilon u_{j}(x), \quad j=0,1,
\end{aligned}
$$

with $L$ from (1.1), and our goal is to replicate the Taniguchi-Tozaki results (Figure 6) for (6.1).

TheOREM 3. Let $Q_{0}$ be the number from (5.6), set $Q_{+}=Q_{0}, Q_{-}=-1-Q_{0}, s_{+}+$ $\beta Q_{+} l_{*}=s_{-}+\beta Q_{-} l_{*}$, and suppose that $\left\lfloor s_{ \pm}\right\rfloor+Q_{ \pm} \geq 0, s_{ \pm} \geq 1$, and

$$
\min \left\{\left\lfloor s_{ \pm}\right\rfloor,\left\lfloor s_{ \pm}\right\rfloor+\beta Q_{ \pm} l_{*}\right\}>\frac{n+2}{2} .
$$

Finally, assume that $w_{j} \in H^{s_{-}-j}\left(\mathbb{R}^{n}\right), j=0,1$, and that $f=f(u)$ is an entire function with $f(0)=f^{\prime}(0)=0$. Then there are (for sufficiently small $\varepsilon>0$ ) unique solutions

$$
u, v \in H^{s_{-}, s_{+}, Q_{-}, Q_{+} ; \lambda}\left((-1,1) \times \mathbb{R}^{n}\right)
$$

to (6.1) and (6.2), respectively. Their difference $u-v$ satisfies

$$
u-v \in H^{s_{-}+\beta, s_{+}+\beta, Q_{-}, Q_{+} ; \lambda}\left((-1,1) \times \mathbb{R}^{n}\right) .
$$

Proof (sketched). Using (5.5) and inverting the time direction, we can obtain an a priori estimate for solutions to a linear equation $L w=f(t, x)$, where $-1 \leq t \leq 1$ :

$$
\begin{aligned}
& \|w\|_{H^{s_{-}, s_{+}, Q_{-}, Q_{+} ; \lambda}\left((-1,1) \times \mathbb{R}^{n}\right)} \\
& \quad \leq C\left(\left\|w_{0}\right\|_{H^{s_{-}\left(\mathbb{R}^{n}\right)}}+\left\|w_{1}\right\|_{H^{s_{-}-1}\left(\mathbb{R}^{n}\right)}+\|f\|_{H^{s_{-}-1, s_{+}-1, Q_{-}+1, Q_{+}+1 ; \lambda}\left((-1,1) \times \mathbb{R}^{n}\right)}\right) .
\end{aligned}
$$

The proof of this estimate runs similar to the proof of Proposition 5.1. This gives us $v \in H^{s_{-}, s_{+}, Q_{-}, Q_{+} ; \lambda}\left((-1,1) \times \mathbb{R}^{n}\right)$. The assumptions on $s_{ \pm}, Q_{ \pm}$imply that $B=$ $H^{s_{-}, s_{+}, Q_{-}, Q_{+} ; \lambda}\left((-1,1) \times \mathbb{R}^{n}\right)$ is an algebra under pointwise multiplication. The superposition $f=f(u)$ maps this edge Sobolev space into itself, since $f$ is entire. From $f^{\prime}(0)=0$ and Proposition 3.6, we then can deduce that $\|f(u)\|_{B} \leq C(R)\|u\|_{B}^{2}$ provided $\|u\|_{B}<R$. Banach's fixed point theorem is applicable if $\varepsilon>0$ is small enough. This proves $u \in H^{s_{-}, s_{+}, Q_{-}, Q_{+} ; \lambda}\left((-1,1) \times \mathbb{R}^{n}\right)$. From Lemma 3.2 it can be concluded that

$$
f(u) \in B \subset H^{s_{-}-1+\beta, s_{+}-1+\beta, Q_{-}+1, Q_{+}+1 ; \lambda}\left((-1,1) \times \mathbb{R}^{n}\right) .
$$

The difference $u-v$ solves $L(u-v)=f(u)$ and has vanishing Cauchy data for $t=-1$. An application of the above mentioned a priori estimate concludes the proof. 


\section{References}

[1] J. Bergh, J. Löfström, Interpolation Spaces. An Introduction, Grundlehren Math. Wiss. 223, Springer, Berlin, 1976.

[2] F. Colombini, E. Jannelli, S. Spagnolo, Well-posedness in the Gevrey classes of the Cauchy problem for a non-strictly hyperbolic equation with coefficients depending on time, Ann. Scuola Norm. Sup. Pisa Cl. Sci. (4) 10 (1983), 291-312.

[3] F. Colombini, S. Spagnolo, An example of a weakly hyperbolic Cauchy problem not well posed in $C^{\infty}$, Acta Math. 148 (1982), 243-253.

[4] P. D'Ancona, M. Di Flaviano, On quasilinear hyperbolic equations with degenerate principal part, Tsukuba J. Math. 22 (1998), 559-574.

[5] M. Dreher, Weakly hyperbolic equations, Sobolev spaces of variable order, and propagation of singularities, Osaka J. Math. 39 (2002), 1-37.

[6] M. Dreher, M. Reissig, Propagation of mild singularities for semilinear weakly hyperbolic equations, J. Anal. Math. 82 (2000), 233-266.

[7] M. Dreher, I. Witt, Edge Sobolev spaces and weakly hyperbolic equations, Ann. Mat. Pura Appl. (4) 180 (2002), 451-482.

[8] Y. Egorov, B.-W. Schulze, Pseudo-Differential Operators, Singularities, Applications, Oper. Theory Adv. Appl. 93, Birkhäuser, Basel, 1997.

[9] V. Ya. Ivrĭ, V. M. PetKov, Necessary conditions for the correctness of the Cauchy problem for non-strictly hyperbolic equations (in Russian), Uspekhi Mat. Nauk 29 (1974), no. 5, 3-70; English transl.: Russian Math. Surveys 29 (1974), no. 5, 1-70.

[10] K. Kajitani, K. Yagdjian, Quasilinear hyperbolic operators with the characteristics of variable multiplicity, Tsukuba J. Math. 22 (1998), 49-85.

[11] O. A. Oleinik, On the Cauchy problem for weakly hyperbolic equations, Comm. Pure Appl. Math. 23 (1970), 569-586.

[12] M.-Y. QI, On the Cauchy problem for a class of hyperbolic equations with initial data on the parabolic degenerating line, Acta Math. Sinica 8 (1958), 521-529.

[13] M. ReIssig, Weakly hyperbolic equations with time degeneracy in Sobolev spaces, Abstr. Appl. Anal. 2 (1997), 239-256.

[14] M. REISsig, K. YagdjIAn, Weakly hyperbolic equations with fast oscillating coefficients, Osaka J. Math. 36 (1999), 437-464.

[15] B.-W. Schulze, Boundary Value Problems and Singular Pseudo-differential Operators, Pure Appl. Math., J. Wiley, Chichester, 1998.

[16] K. Taniguchi, Y. TOZAKI, A hyperbolic equation with double characteristics which has a solution with branching singularities, Math. Japon. 25 (1980), 279-300. 